\title{
Occipital plagiocephaly: a critical review of the literature
}

\section{Harold L. Rekate, M.D.}

Pediatric Neurosurgery, Barrow Neurologic Institute, Phoenix, Arizona

The objective of this review was to determine what information is available on the incidence, pathophysiology, late complications, and treatment paradigms for occipital plagiocephaly based on a critical review of the literature obtained from recognized databases in peer-reviewed scientific publications.

The content of this article is based on a critical review of the literature, and when discussing treatment options, classification of those articles with respect to the strength of the recommendations they contain.

Using standard computerized search techniques, databases containing medical literature were queried for key words related to occipital plagiocephaly beginning in 1966. Key words used for this search were: lambdoid, craniosynostosis, cranial sutures, facial asymmetry, torticollis, and plagiocephaly. Titles of all articles were scanned for relevance. Copies of all potentially relevant articles published in the English language were obtained and received at least a cursory review. Several articles not captured by these methods were found to be important when referenced in the articles obtained. Articles discussing treatment were divided into Class I, Class II, and Class III data for the purpose of deciding on their applicability to the development of a potential consensus for the treatment of this controversial condition.

Using the aforementioned key words, there were 4308 articles identified with potential relevance: scanning by title excluded all but 89 . Of the 89 , those with on-line abstracts were scanned, the remainder were obtained via interlibrary loan when needed for scanning of the article itself. The actual incidence of occipital plagiocephaly is unknown and there are no population-based studies of its incidence or prevalence. The reported incidence of lambdoid craniosynostosis ranges from 3 to $20 \%$ with the differences primarily due to differences in diagnostic criteria. With the possible exception of a lambdoid suture, which is replaced throughout its entire course by a dense ridge of bone, there are no other diagnostic criteria upon which there is agreement. There are no Class I and only one Class II studies in which a group of patients treated with one form of therapy is compared with another form of therapy or an untreated group. Treatment options that are recommended include observation only, mechanical treatments such as exercises, positioning, remodeling helmets, and a wide variety of surgical techniques. Very few reports accessed through the aforestated methodology report patients suffering any significant late effects of occipital plagiocephaly, although it may be morphometrically evident in as many as $14 \%$ of adults.

Controlled clinical trials will be needed before any form of intervention can be generally recommended. If surgery, which is expensive and potentially dangerous, is to continue to play a role in the management of this condition, efforts should be made to ascertain from the general population which patients who 
have not been treated have suffered from this lack of treatment.

\section{Key Words * lambdoid * craniosynostosis * cranial sutures * facial asymmetry * torticollis * plagiocephaly}

As can be easily discerned from a perusal of the other articles presented in this issue of Neurosurgical Focus, the management of babies with misshapen heads is controversial. This very common condition has been called by many names including "benign positional molding," lambdoid plagiocephaly," "deformational plagiocephaly," and "occipital or posterior plagiocephaly." Occipital plagiocephaly (OP) is most frequently caused by deformational forces that occur before and after birth but which may be due to true lambdoid craniosynostosis. Referrals for evaluation and treatment of this condition have increased dramatically. Dias and coworkers[4,5] have reported that this condition is second only to hydrocephalus as a reason to be seen in the pediatric neursosurgical unit in Buffalo. At the Barrow Neurological Institute in Phoenix we see between three and five new patients with this condition per week.[27] The families of many of these babies have been prepared for emergency surgery for protection of neurological function or vision. When second opinions are sought, the families become distraught by the extreme differences in approach advocated by different specialists in the same field. Third-party payors remain confused; they are often willing to pay for craniofacial procedures but will not even consider the possibility of paying for orthotic devices that may also be effective at essentially no risk and considerably less cost.

To properly evaluate new information regarding the pathophysiology, diagnostic criteria, and treatment options as it becomes available, it seems appropriate to look critically at what has been published to date. This will allow the new work to be put into the context of what has gone before and to suggest ways in which further work may lead to reliable information on which to make treatment decisions. The purpose of this review is to attempt to ascertain what is known about OP: its incidence with and without lambdoid craniosynostosis, its pathophysiology, the potential for late complications with and without treatment, and what form treatment should take. Are any forms of treatment distinctly better than others? Finally, what would it take to develop a practice guideline for the management of this form of misshapen head?

\section{REVIEW}

In performing this review of the available medical literature, an attempt was made to use the techniques developed by the head injury guidelines task force in the production of their publication.[18] A computerized search of the medical literature available from standardized medical databases of peer-reviewed articles dating back to 1966 was performed. The key words used for this search were: lambdoid, craniosynostosis, cranial sutures, plagiocephaly, facial asymmetry, and torticollis. A list of 4308 articles was generated and they were scanned by title. A total of 89 articles were found to be of potential interest. Of these, 52 articles had been captured by the key word "plagiocephaly." A printout of the references, including abstracts, was made to allow further refinement of the list. Articles of interest were obtained from the medical library at St. Joseph's Hospital and Medical Center or by interlibrary request. Several references that were not captured by this methodology but seemingly of importance were discovered through cites within the articles read for this review.

The most difficult part of the search process was the confusion brought on by the failure among authors to agree on the definition of terms. Until very recently, the majority of articles published in the neurosurgical literature equated the term "plagiocephaly" with unilateral coronal craniosynostosis. This 
condition is not being discussed in this review. Articles written primarily for pediatricians used the term "plagiocephaly" to mean the parallelogram shape of the head that we have come to associate with OP. These terms are not defined in articles written by ophthalmologists, dentists, and dysmorphologists, which usually means that unilateral coronal craniosynostosis is being discussed but often the type or cause of plagiocephaly is not specified and the reader must attempt to discern which of the entities is actually meant. The first finding of this review is that there is a great need for a uniformity of nomenclature in this literature.

Each article was scrutinized for content and, if appropriate, assigned as relevant to one or more of the following categories: incidence, diagnostic techniques, late complications, treatment, and pathophysiology. Those assigned to the treatment category were subdivided among the various types of treatment advocated, including observation, repositioning and exercises, helmet therapy, and surgery. Each of those in which a certain treatment was evaluated or discussed was subjected to the scrutiny of categorization as Class I, Class II, or Class III.[18] Class I evidence: prospective randomized controlled trials (PRCT); Class II evidence: clinical sudies in which the data were collected prospectively and retrospective analyses which were based on clearly reliable data; and Class III evidence: most studies based on retrospectively collected data.

Other areas such as incidence, in which the above categorization is not applicable, were reviewed to determine experimental and statistical design and these were compared with other articles in the same category to assess potential usefulness.

\section{RESULTS}

\section{Incidence}

Ten articles reporting the incidence of OP or lambdoid craniosynostosis were identified. Two of these studies[10,31] are population-based studies and compare their results to those that had been previously published. The first of these studies[31] reports a 5.5\% incidence of lambdoid craniosynostosis in a population of babies born with craniosynostosis of any kind. The general incidence of craniosynostosis in their report was six cases in 10,000 live births. The incidence of lambdoid craniosynostosis was similar to but somewhat higher than that reported previous to that study. The second of these population-based studies performed in Olmested County, Minnesota, which found an incidence of confirmed cases of craniosynostosis to be 3.1 cases per 10,000 births and 13.6 suspected cases per 10,000 births.[10] Based on these studies, plagiocephaly caused by isolated lambdoid craniosynostosis should occur approximately three times in 100,000 births (approximately 100 times less frequently than myelomeningocele).

Muakkassa, et al.,[25] reviewed 74 patients thought to have premature closure of the lambdoid suture from a total population of 404 patients managed for craniosynostosis at the Hospital for Sick Children in Toronto between 1972 and 1984. This would yield an 18\% incidence of lambdoid craniosynostosis when compared to the data presented above.[10,31] The difference seems to lie in the definition of lambdoid craniosynostosis. In only three of the 74 patients was the suture found to be pathologically fused and it is probably only that group which was addressed in the studies quoted above. Most if not all of the remaining 71 patients described in the study probably suffered from OP. Recalculations based on the above conclusion reveal that pathologically distinct lambdoid craniosynostosis occurs in three patients in a population of 333 children with craniosynostosis representing less than $1 \%$ of craniosynostosis treated at that institution. 
Dunn[7] reported an incidence of one in 300 births of OP due to sternomastoid torticollis. He postulates that both are due to in utero constraint. Subsequent articles by Clarren and coworkers[2,3] quote this figure as well; I have been unable to find population-based studies from which incidence data can be derived. Clarren and coworkers, [2,3] after quoting Dunn[7] on the incidence data, state that approximately $10 \%$ of these children may be left with permanent disfigurement so as to define a population in whom intervention may be justified. Neither a numerator nor a denominator is given for this number making the actual percentage of the population affected by this condition, for whom treatment may be indicated, essentially unknown. Plagiocephaly may be a very common finding in otherwise normal populations of patients who are totally unaware that there are any problems at all. Watson[36] studied a large number of patients with congenital disorders that were highly associated with plagiocephaly, including congenital hip dislocation, "bat ears," congenital scoliosis, and sternomastoid tumors.[36] Using a self-constructed apparatus of articulated rulers he showed objectively quantifiable plagiocephaly in $60 \%$ of infants with congenital hip dislocation which decreased to $32 \%$ by adolescence. He also examined a total of 485 normal control patients. His findings are fascinating. Based on the measurement techniques that he advocates, he found significant degrees of asymmetry in $48 \%$ of normal babies less than 1 year of age. He was able to show that $14 \%$ of normal adult subjects (including some of the parents of the children with congenital hip dislocation) had significant skull asymmetry (plagiocephaly).[36]

Finally, the question arises as to the effect of the new recommendations of the American Academy of Pediatrics as it relates to positioning of the sleeping child to prevent the sudden infant death syndrome. Many oral arguments and at least one published report[19] have suggested that the recent increases in the referral of patients with OP are due to a dramatic increase in its incidence, which has resulted because of the change from prone to supine positioning of most newborns, based on the Academy's recommendations. Because OP will be shown below to be primarily a mechanical problem caused by positioning and because most children diagnosed with OP prior to these recommendations had a history of intolerance to prone sleeping, this assumption seemed logical. Kane and colleagues[19] reported a sixfold increase in the annual number of referrals to their craniofacial center beginning in 1992 , the year of the Academy's task force report, as compared with the previous 13 years. Commentary subsequently published in The Journal of Pediatrics[15] argued, by conducting an analysis of the actual changes in sleeping position that occurred slowly following the report, that the increase in referrals to that center could not be explained (at least completely) on the basis of these recommendations and suggested that greater physician and public awareness of the condition played a major role in the changes in referral numbers.

The incidence of OP must be very high; however, the actual numbers are not known. The often-quoted incidence of one in 300 births probably represents a minimal number and reflects the subjectivity in the definition of this condition and the variations in the threshold for making the diagnosis. Before attempting to study the actual incidence of this condition in a population, a concensus must be reached on the diagnostic criteria to be used. By measurement, this condition must be common in the adult population as well.[36] To establish principles of treatment in babies with this condition, it is important to identify the effects of nontreatment in the adult population of affected individuals.

The incidence of isolated unilateral lambdoid craniosynostosis is very low, with estimates ranging from less than $1 \%$ to approximately $5 \%$ of patients, implying that the population-based incidence should be less than three cases per 100,000 births. Estimates of the incidence of lambdoid craniosynostosis 
indicating as much as $20 \%$ of crainosynostosis being due to a unilateral involvement of the lambdoid suture are almost certainly obtained using radiographic criteria that include a majority of patients who have OP. It is essential to clarify these issues so that subsequent articles clearly distinguish between the specific case of lambdoid craniosynostosis and OP.

\section{Pathophysiology}

If the controversy regarding what is and what is not lambdoid craniosynostosis is excluded there is general agreement among authors as to the pathophysiological mechanism of OP. The problem results from an external deforming force applied consistently over a long period of time to a specific region of the infant's head. There are both prenatal and postnatal factors involved in the distortion and some conditions that have been recognized to predispose to this condition.

The article by Watson[36] already mentioned in the section on incidence reviews 214 children referred for evaluation who had OP accompanying the presenting complaints. Children were of all ages. The conditions associated with plagiocephaly included congenital hip dislocation in 107 children, bat ears in 17, congenital scoliosis in 48, and sternomastoid "tumors" in 42. Bat ears are ear deformities in which the involved ear is much more prominent and to some extent deformed but can be manually made to return to a more normal position. This appearance of the ear, which is on the flattened side, may simply reflect the forward and downward position of the ear due to the cranial deformity. Both congenital hip dislocation and bat ears are recognized to be caused by prenatal fetal constraint and are more common in the context of oligohydramnios and multiple births. These two associations suggest that plagiocephaly results from prenatal factors leading to constraint of the head in the maternal pelvis. On the other hand, congenital scoliosis and sternomastoid tumor result from constant head turning in the same direction. Although the problem may exist prenatally, these conditions are more likely to result in significant plagiocephaly postnatally, particularly if the child sleeps supine. Eleven of the 48 patients with congenital scoliosis had distinct vertebral body anomalies that resulted in constant head rotation. It should be noted that in Watson's report he describes the side of the plagiocephaly as the posteriorly displaced side with the flattened forehead and bulging occiput. He has therefore found a 2:1 left/right ratio of plagiocephaly. This nomenclature is the opposite of that which names of the side of plagiocephaly for the area of the occiput that is being distorted. When this is taken into consideration the incidence of side-to-side preference agrees with most contemporary authors who find a consistent 2:1 right/left preference.

Congenital muscular torticollis is an associated phenomenon reported by many authors to be a causative factor in the development of plagiocephaly. Often the degree of the torticollis is rather mild and is exhibited as a preference for one sidedness. Slate and colleagues[32] evaluated 30 babies for congenital muscular torticollis of whom 26 had OP. Half of these patients (13 of 26) were shown to have rotatory subluxation of C-1 on C-2 by computerized tomography (CT) scan. None of these patients required cervical fusion, although seven of the 26 required neck muscle surgery to improve the plagiocephaly. This rotational difficulty may begin as a prenatal event but its continuing presence may be responsible for the increase in severity of the OP during the 1st year of life.

With the exception of patients who have severe congenital anomalies of the spine which lead to severe mechanical scoliosis, the most severe form of congenital muscular torticollis is caused by an entity known as the sternomastoid or sternocleidomastoid "tumor" of infancy (SMT).[33,38] Babies and children with this condition exhibit a severe degree of torticollis that may persist into adulthood and is 
associated in a high percentage of patients with severe degrees of OP. An actual mass is palpable in the muscle belly itself. When resected the mass is hypertrophic scar tissue and it is suggested that SMT is due to venous infarction of the muscle caused by intrauterine constraint. Sternocleidomastiod tumor usually responds to physical measures such as stretching exercises but occasionally it is so severe that removal of the mass and lysis of the muscle is required. Surgery of the neck muscles usually leads to improvement of the OP.[33] When the severe degrees of torticollis associated with SMT or congenital spinal deformity are left untreated during childhood, facial deformity may be severe enough to be a problem in adolescence and adulthood. Ferguson[6,8] reported three patients with severe torticollis and OP in whom he recommended surgical management of the torticollis followed by physical therapy and, ultimately, surgical correction of the facial asymmetry with maxillary osteotomies. It should be emphasized that the three new patients managed surgically by Ferguson and the five previous patients cited in this article all had severe, obvious cervical scoliosis that remained untreated into adulthood and that these eight patients are the only individuals who have been identified by the search methodology used here to have required treatment in later life for facial asymmetry due to OP.

Other conditions associated with OP include patients with asymmetric brain injuries or developmental abnormalities evidenced by severe asymmetric spasticity or major motor epilepsy occurring unilaterally[34] and intentional skull deformity.[4,5,11]

Strabismus, especially involving vertical eye movements, is common in the more severe forms of this disorder but is more likely to result from than cause OP.[1,9,12,21,22,35,37] Strabismus secondary to congenital superior oblique paresis leads to a head tilt in the first 6 to 12 months of life and may lead to secondary facial asymmetry.[12] Traumatic superior oblique palsies, however, do not lead to secondary facial deformity. Goodman and colleagues[12] postulate that early correction of strabismus secondary to congenital weakness of the superior oblique muscle may improve the resulting facial asymmetry but they also suggest that positioning and neck stretching exercises are probably more important. No older children or adults in this series required intervention for their facial deformities.

In keeping with the theme of prenatal restriction of skull movement and the resulting pressure on and distortion of the skull, a high percentage of babies with OP will have heads that are large for their gestational age or are frankly macrocephalic. Sawin and colleagues[30] have studied 31 infants with OP and compared the results found on CT scanning with 20 normal controls. Twenty-nine of the 31 patients with OP exhibited the generalized increase in subarachnoid cerebrospinal fluid associated with macrocephaly and termed "external hydrocephalus." Whether the authors suggestion that the presence of this excess of subarachnoid fluid predisposes to calvarial distortion because the ability to shift the fluid creates an increase in the plasticity of the skull must remain conjecture at this point.[30] It is also possible that the larger the head the more likely the child is to have external hydrocephalus and also, independently, is more likely to suffer from plagiocephaly as a result of in-utero constraint. The idea of increased plasticity with water underlying the area of skull deformity is intriguing.

Occipital plagiocephaly is associated with a great many conditions leading to a unifying pathophysiology. These infants either due to their external environment or to intrinsic abnormalities of their posture (SMT, cervical spine anomalies, asymmetric tone) lie in the same position for many hours over many days with an area of the skull being distorted by a hard, flat surface. Prenatally this surface is the maternal hip and postnatally it is the crib mattress. Just as when a baby with massive hydrocephalus undergoes ventriculoperitoneal shunting, causing a severe degree of scaphocephaly, persistent deformity in the area of a suture in which the two edges of the suture are chronically forced together may also occur 
in the lambdoid suture, leading to the radiographic evidence of lambdoid craniosynostosis in patients who would otherwise be diagnosed with OP.

\section{Diagnostic Studies}

In contrast to the issues related to the pathophysiological mechanisms of OP in which there is general agreement, diagnostic testing and interpretation are matters of great debate, with little or no agreement among the various authors and investigators.

As we endeavor to discuss treatment options, two different philosophical approaches to the treatment of OP must be distinguished. The classic neurosurgical teaching is that craniosynostosis is a progressive disease with increase in skull deformity being seen as the brain grows. One of the main goals of therapy is to prevent worsening of the configuration. In this paradigm, the accurate diagnosis of craniosynostosis becomes critical. The clinical and radiographic criteria for the diagnosis of true lambdoid craniosynostosis have not been established. Some authors[5,14,25,27] have thought that there was something different about this condition that distinguished it from other forms of craniosynostosis, and I have fallen into that trap myself.[27] With this understanding or misunderstanding, a small percentage of patients reported will actually have radiographic or pathological evidence of sutural closure with the usually palpable or visual ridging that occurs in this condition. At surgery the sutures will often show fusion with the underlying dura that is absent in other forms of craniosynostosis. True lambdoid craniosynostosis is exceedingly rare. It produces a skull that does not appear to be a parallelogram, as in postural OP, but rather the side of occipital flattening is ipsilateral to the forehead flattening which creates a rhomboid rather than the usually found parallelogram. There is also a prominent ridge that can easily be palpated in the area of the mastoid bone or seen on CT scanning.[16] Unless these features are found the patient should not be treated to prevent further worsening of the situation, and the radiographic investigations to diagnose true lambdoid synostosis are entirely justified.

The second approach to the patient with OP does not depend on the patient's having or not having craniosynostosis but relies on the degree of the patient's functional and cosmetic deformity in making the decision whether to treat the deformity.[4,5] In this situation distinguishing between lambdoid craniosynostosis and postural OP loses its significance and the importance of diagnostic studies devolves to studying the results of treatment and comparing one patient to another or, hopefully, one form of treatment to another.

In a paper written and published before the advent of CT scanning, Watson[36] advocates the use of a set of articulated rulers to judge the degree of asymmetry of the skull. His method is quite simple and it can pick up subtle asymmetries both in an obviously affected group of individuals and in a normal control population. The simplicity and lack of expense in using this technique are quite appealing but no data are presented as to interrater reliability or reliabiltity of the measurements made at different times by the same examiner. It is a technique that may be effected by the experiences or biases of the observer. This technique may prove useful in decreasing the cost of long-term follow-up studies if such data becomes available.

Sophisticated technological procedures have been advocated for the diagnosis of various forms of plagiocephaly. Glat and colleagues[11] have developed a computer analysis in three dimensions of measurements taken from CT scans. From this they are able to discern three distinct types of plagiocephaly: synostotic plagiocephaly, deformational plagiocephaly, and plagiocephaly associated with hemifacial microsomia. This paper is very preliminary and should be considered work in progress. The 
authors' hope is to be able to use this complicated technique to establish objective criteria for intervention in these cases, an admirable goal.

Lo and colleagues[23] have studied the changes at the skull base as seen on three-dimensional reconstructions of CT scans. One interpretation of the data presented in their paper is that true synostosis, whether it be coronal or lambdoid, will produce changes throughout the skull base with rotation of the skull base elements around an axis at the center of the skull base. This center is situated centrally in the quadrilateral space bounded by the anterior and posterior clinoid processes. In case of external deformation such as OP, the calvaria, but not the central skull base, rotates around this axis. If this hypothesis is correct and it is certainly supported by the data they present, a line drawn down the crista galli and passing through the midpoint of the skull base as described above will not pass through the center of the foramen magnum when the cause of the distortion is actual craniosynostosis and may miss it altogether. In cases of postural skull distortion, however, only the calvaria and the most lateral rays of the sphenoid and petrous bones will be displaced and the above line will pass near the center of the foramen magnum. The investigators have developed mathematical descriptions of these findings that will result in a great deal of concurrence among individual radiologists who read the radiographs. To be totally objective in reviewing this paper it would be essential to know that the four patients who they have defined as having unilateral lambdoid craniosynostosis met the criteria for that diagnosis described above.[16] As opposed to the complicated techniques described in the article by Glat, et al.,[11] the principles outlined here are simple and easy to follow and should not differ significantly from one center to another. Although these techniques may be useful in deciding whether true synostosis exists, they do not appear to be useful in differentiating the degree of severity of OP from one patient to the next or in a single patient before and after treatment.

Formal measurement of standard anthropomorphic lines should prove useful in the assessment of the severity of OP. $[24,28]$ There is a learning curve in the use of these callipers and there is poor interobserver concurrence until all observers have significant experience in making these measurements. Comparisons of such measurements when done by experienced observers will be a reasonably reliable and inexpensive method to compare the severity of OP from one patient to another and before and after treatment.

As stated in a commentary in Pediatrics[15] and an editorial in Lancet,[20] the best diagnostic test in a case of occipital plagiocephaly is the physical examination. The examination requires that the physician or nurse practitioner look down at the top of the baby's head. The asymmetry of the ear position and flatness of the occiput are clearly visible by this simple and inexpensive technique that can be done quickly. If the diagnosis is made in the 1 st week of life and mechanical means are instituted to counteract the distortional effects, the question of whether to intervene and how will become moot.[15,20]

\section{Late Effects}

Occipital plagiocephaly is a common problem in the newborn period and there is no reason to believe that the problem has not existed throughout time. Except for the management of a small number of patients with overt lambdoid craniosynostosis, neurosurgeons played a small or no role in the management of patients with this condition until quite recently.[14,25] To judge the importance and effects of intervention, whether it be mechanical or surgical, some attempt should be made to understand the natural history of the untreated condition. This should not be difficult because before the late 1970s very few babies were treated with anything except reassurance of their parents or occasionally lysis of 
the sternocleidomastoid muscle.[6,33,38] There should be a very large number of adults who have residual evidence of untreated OP.[36] Studying the negative effects suffered by some of these adults should help to define the risk involved in nontreatment. For this reason, a concerted effort was made to find articles in which adolescents and adults with untreated OP were seen later in life.

Adults do have OP. Watson[36] studied the relationship of OP to conditions such as congenital hip dislocation and congenital scoliosis. In this study, although OP was present in half of the babies under 1 year of age who manifested these problems, it could only be discerned by his measuring techniques in $14 \%$ of adolescents with these conditions, suggesting that there was a significant tendency for OP to improve over time. He also studied 485 normal adults, some of whom were the parents of the affected individuals. He was able to find significant asymmetry in $14 \%$ of normal adults.

Anterior plagiocephaly (unilateral coronal craniosynostosis) is well known to produce significant abnormalities of binocular eye movements. Limon de Brown and colleagues[22] studied the relationship between plagiocephaly and strabismus. From the pictures supplied, it is clear that the moderate-to-severe cases were representative of untreated unilateral coronal synostosis. It is likely that the six patients whom they indicate have minimal deformation have OP. Although the total number of patients screened is not mentioned and the techniques are not thoroughly described, anthropometric measurements were obtained in a large number of patients with strabismus. Considering that their screening uncovered 13 cases of moderate-to-severe orbital dystopia (untreated coronal craniosynostosis), the total number of patients screened must have been very large. Six patients were found to have "minimal" plagiocephaly by anthropometric measurements. Although these patients did demonstrate strabismus, all of them had normal visual acuity and binocular vision in primary position. None of these patients were aware of their asymmetric heads. The suggestion is made that minor degrees of OP may cause strabismus because of the asymmetry. Because the total number of patients screened is not known, it is not known whether this represents the coexistence of two independent common diseases or is somehow cause and effect. The effect on the patient's life was minimal and the problems were found only by compulsive screening techniques.[22]

A single study of patients compares the effects on ocular motility and strabismus of synostotic and deformational frontal plagiocephaly.[6,8] Deformational frontal plagiocephaly seems to be another synonym for the more severe forms of OP that lead to compensatory changes in the forehead and face. Fredrick and colleagues[9] studied 13 patients with deformational frontal plagiocephaly and found that one had horizontal strabismus not associated with the geometry of the head tilt and therefore considered it to be an incidental finding.

Facial asymmetry is known to lead to significant problems in dental management, especially orthodontia, and to create difficulties with the temporomandibular joint. Cross-referencing plagiocephaly with facial asymmetry yielded one article that related a problem caused by plagiocephaly from an oral surgical point of view. This case report is of a woman who's friend noticed that one side of her face was swollen but not painful. The patient was not aware of the problem until it was pointed out to her.[29] An extensive workup revealed no abnormalities except a previously unrecognized plagiocephaly. Early photographs of the patient confirmed this as having existed since early childhood.[29]

Facial asymmetry can be found in a relatively large number of adult patients if it is rigorously investigated. Plagiocephaly severe enough to be acknowleged by the patient is exceedingly rare, as judged by the search methodology used here. The need for treatment of OP is found only in patients with 
overt and untreated congenital muscular torticollis and cases fulfilling these criteria are so rare as to warrant careful study and publication.

There are several caveats here. Although the theoretical problems of visual disturbances and orthodontic/oral surgical difficulties have not been documented using the methodologies reported here, they may exist but perhaps they have not been reported. Failure to identify them may be due to a lack familiarity with the "words of art" from those specialties and thus the articles have been published but have eluded the search. To test this hypothesis, I have discussed the problem of late effects of OP in adults with a number of professionals who may have reason to encounter these patients in adulthood. These interviews were conducted unscientifically and the information simply observational. Among pediatric ophthalmologists, orthodontists, oral surgeons, and barbers/hair stylists, OP was not recognized as a problem with which they were required to deal. The only group that showed significant awareness were the oculists because they frequently needed to adjust the glasses so that one ear piece was shorter than the other. Further attempts should probably be made to search at least the dental literature to ascertain whether untreated patients with this condition require special attention later in life.

The second problem not addressed by this review is psychosocial. When discussing the need for intervention in patients with abnormal head shapes of whatever cause, the effect of being perceived as abnormal by one's peers, especially in adolescence, is always a compelling reason to consider intervention. Based on this literature search, it is unlikely that this effect has been studied in the context of OP. Parenthetically, with extensive searching and advertising to rather large groups, I have identified two adult males with severe residual OP and interviewed them. Despite the severity of the condition and although both of the affected individuals were aware that they had the abnormality, neither had felt self-conscious about the situation prior to the interview and neither was ever teased because of the shape of the head. If aggressive intervention is to be recommended, a search should be made for adolescents and adults with OP to determine what effect if any it has had on their lives.

The possibility that cranial distortion without increased intracranial pressure or definable damage being done to the distorted underlying areas of the brain could lead to overt or subtle neuropsychological problems has not been reported to this point.

\section{Treatment Options}

Three categories of treatment are available for OP: 1) expectant/observation/reassurance with or without minor mechanical intervention; 2) aggressive mechanical intervention with or without cranial banding; and 3) surgical. Until very recently and conceivably even now, by far the greatest number of babies with OP either have gone unrecognized or have been treated expectantly, usually with reassurance of the mother and frequently with instructions to the family to prop the baby so that the bulging side of the head is presented to the crib mattress. There are no outcome studies that follow patients treated in this way and there are no population-based incidence studies that evaluate the natural history of the condition without specific intervention.

Aggressive mechanical intervention involves an active diagnosis of OP being made and intervention intended to reverse the forces that have distorted the head. Interventions include neck stretching exercises, with or without the supervision of a physical therapist, and active attempts to modify the child's sleeping position. There is one report in which this approach was taken (Class III study) that showed measurable improvement in cranial asymmetry using this form of intervention alone.[13] For the most part, aggressive mechanical intervention now refers to the use of the cranial remodeling helmet or 
dynamic orthotic cranioplasty. The original descriptions of the use of helmet therapy for the treatment of OP were reported by Clarren and coworkers. [2,3] These two studies overlap to some extent with that of the four patients treated and reported in the 1979 article who were also included in the 28 patients treated and reported in the second follow-up study. The 1981 article does compare, using a very subjective scoring scale, the outcome in the 25 patients who completed treatment with those who did not complete the treatment or refused it. In this scale $2+$ indicated normal appearance, $1+$ indicated improvement but not to normal, and 0 indicated no improvement. Nineteen of 25 patients treated achieved a normal appearance, and the remaining six were improved to some degree. Of patients who failed to complete or refused treatments, seven were unimproved and six experienced a modest improvement. The reader is not told at what point or by whom these assessments were made. This second study qualifies as a Class II study, and although the methodology is not ideal, it gives significant support to the use of helmets to correct deformational abnormalities of the skull.[2]

A recent study by Ripley and colleagues[28] describes the use of helmet therapy with dynamic orthotic cranioplasty band in 124 infants and children who were followed over time with extensive anthropometric measurements. Using these measurments, significant improvement was noted in the anthropometrically measured asymmetry in all but a few patients. They also noted that the corrections were maintained after use of the band was discontinued but that no further improvement could be observed in these patients. The authors state that OP does not improve without this type of intervention but they do not supply any data from their center to validate the statement. From the point of view of making treatment decisions, this study contains Class III information because it does not compare the results to concurrent or historical controls.

In the article by Moss[24] that accompanies the present review in this issue of Neurosurgical Focus, measurements similar to those made by Ripley, et. al.,[28] were made in patients with OP who were treated without the band. This review was not available from the literature search and thus was technically not available for this review, but the finding that is in agreement with the article by Hellbusch, et al.,[13] discussed above is that mechanical treatment without the use of the band may be useful in the management of OP.

Surgery for OP is still being advocated in a number of centers. Forms of treatment advocated range from unilateral strip craniectomy $[4,5,14,25]$ to extensive bilateral occipital cranial remodeling techniques in which the entire torcula and both transverse sinuses are exposed.[17,26] Three of these studies are composed of Class III evidence and all rely on completely subjective assessments in the early postoperative period in establishing success. Dias and colleagues[4,5] studied 30 infants who had undergone strip craniectomy of the lambdoid suture for OP, and they attempted to obtain objective information about the degree of asymmetry from measurements taken from CT scans. They were able to obtain late posttreatment CT scans taken 3 months to 4.2 years following the surgery in 18 of the patients. Significant improvement was obtained in terms of these measurements but there remained significant differences on the scan between the group undergoing surgery and the control group of normal individuals. This study also represents Class III information because the results are not compared with untreated controls or patients treated by alternative methods.

Summary of Treatment Options. A) Standards: there are insufficient data to support a treatment standard for OP. B) Guidelines: 1) Mechanical means such as propping of the child and neck stretching exercises are useful in preventing worsening of OP and may help reverse some of the deformity; 2) In patients with moderate-to-severe degrees of OP, helmet or band therapy will result in improvement in the observed 
and measurable asymmetry. The earlier it is applied the more quickly and more completely the correction can be accomplished. The degree of deformity necessary to require the use of this form of treatment cannot be determined from currently available studies; 3) There are insufficient data to support guidelines for the surgical management of OP. C) Options: 1) Neck stretching exercises and lateral propping of the baby's head may be sufficient to correct OP if they are begun early in life and performed consistently; 2) Head band or helmet therapy should be utilized in babies in whom the asymmetry persists despite other mechanical forms of intervention; 3) Head band or helmet therapy should be utilized prior to considering surgical intervention in patients in whom the condition is recognized within the first year of life; 4) For patients with severe residual deformity following head band or helmet therapy or for those in whom referral occurs beyond one year surgical intervention may be considered; 5) If surgery is to be performed for OP there are several techniques which have been reported to be effective. There are no studies showing compelling reasons to chose one over another.

\section{CONCLUSIONS}

Occipital plagiocephaly, which is also called benign positional molding, is a condition that has always been common in infants but rarely recognized in adults. The actual prevalence of this condition in both babies and adults is a subject of conjecture. This condition is being recognized much more frequently now as a result of increased awareness by primary care physicians and the public at large. Current recommendations of the American Academy of Pediatrics that require babies to sleep supine to decrease the incidence of sudden infant death syndrome have played a minor role in the increased frequency of referral of infants to craniofacial and pediatric neurosurgical specialists.

Forms of therapy available to treat this condition include mechanical reversal of the deforming forces, mechanical redirection of those forces using helmet therapy, and surgical intervention. Because little information exists about the true risk of leaving this condition untreated, careful consideration is necessary in determining the role of each of these modalities in the management of the patient with OP.

Recent commentary in Pediatrics by Hunt and Puczynski[15] recommends that primary care physicians and parents take on the responsibility for the diagnosis and management of these children. If the diagnosis can be made in the first few weeks of life and specific strategies attempted to prevent further cranial distortion "orthotic devices such as soft helmets or rigid head bands should rarely, if ever, be necessary, and surgery for PWS [plagiocephaly without synostosis] should never be necessary."[15] At this time this is conjecture, but does represent a testable hypothesis that could well be answered in a short period of time by a well-designed case control study.

\section{References}

1. Bellizzi C, Lamorgese T, Grossi T: Torticolis oculaire: diagnostic et pronostic. J Fr Ophthalmol 6:589-591, 1983

2. Clarren SK: Plagiocephaly and torticollis: etiology, natural history, and helmet treatment. J Pediatrics 98:92-95, 1981

3. Clarren SK, Smith DW, Hanson JW: Helmet treatment for plagiocephaly and congenital muscular torticollis. J Pediatrics 94:43-46, 1979

4. Dias MS, Klein DM: Occipital plagiocephaly: deformation or lambdoid synostosis? II. A unifying 
theory regarding pathogenesis. Pediatr Neurosurg 24:69-73, 1996

5. Dias MS, Klein DM, Blackstrom JW: Occipital plagiocephaly: deformation or lambdoid synostosis? I. Morphometric analysis and results of unilateral lambdoid craniectomy. Pediatr Neurosurg 24:61-68, 1996

6. Dunn PM: Congenital postural deformities. Br Med Bull 32:71-76, 1976

7. Ferguson JW: Cephalometric interpretation and assessment of facial asymmetry secondary to congenital torticollis. The significance of crania base reference lines. Int J Oral Maxillofac Surg 22:7-10, 1993

8. Ferguson JW: Surgical correction of the facial deformitites secondary to untreated congenital muscular torticollis. J Craniomaxillofac Surg 21:137-142, 1993

9. Fredrick DR, Milliken JB, Robb RM: Ocular manifestations of deformational frontal plagiocephaly. J Pediatr Ophthalmol Strab 30:92-95, 1993

10. French LR, Jackson IT, Melton LJ III: A population-based study of craniosynostosis. J Clin Epidemiol 43:69-73, 1990

11. Glat PM, Freund RM, Spector JA, et al: A classification of plagiocephaly utilizing a three-dimensional computer analysis of cranial base landmarks. Ann Plast Surg 36:469-474, 1996

12. Goodman CR, Chabner E, Guyton DL: Should early strabismus surgery be performed for ocular torticollis to prevent facial asymmetry? J Pediatr Ophthalmol Strab 32:162-166, 1995

13. Hellbusch JL, Hellbusch LC, Bruneteau RJ: Active counter-positioning treatment of deformational occipital plagiocephaly. Nebr Med J 80:344-349, 1995

14. Hinton DR, Becker LE, Muakkassa KL, et al: Lambdoid synostosis. Part 1. The lambdoid suture: normal development and pathology of "synostosis." J Neurosurg 61:333-339, 1984

15. Hunt CE, Puczynski MS: Does supine sleeping cause asymmetric heads? Pediatrics 98:127-129, 1996

16. Huang MHS, Gruss JS, Clarren SK, et al: The differential diagnosis of posterior plagiocephaly: true lambdoid synostosis versus positional molding. Plast Reconstruct Surg 98:765-774, 1996

17. Jimenez DF, Barone CM: The Sunrise Technique: the correction of occipital plagiocephaly using bandeau occipital plate and radial osteotomies. Pediatr Neurosurg 22:162-166, 1995

18. Joint Section on Trauma and Critical Care of the American Association of Neurological Surgeons and the Brain Trauma Foundation: Guidelines for the Management of Severe Head Injury. Park Ridge, Ill: AANS, 1995

19. Kane AA, Mitchell LE, Craven KP, et al: Observations on a recent increase in plagiocephaly without synostosis. Pediatrics 97:877-885, 1996

20. The Lancet: Plagiocephaly and torticollis in young infants. Lancet 4:789-790, 1986

21. Lavenent F, Quere MA, Duguet V, et al: [Cogenital paralysis of the IVth nerve and craniofacial 
asymmetry.] Ophthalmologie 1:339-341, 1987 (Fr)

22. Limon de Brown E, Ortiz Monasterio F, Feldman MS: Strabismus in plagiocephaly. J Pediatr Ophthal Strab 25:180-190, 1988

23. Lo LJ, Marsh JL, Pilgram TK, et al: Plagiocephaly: differential diagnosis based on endocranial morphology. Plast Reconstr Surg 97:282-291, 1996

24. Moss D: Nonsurgical, nonorthotic treatment of occipital plagiocephaly: what is the natural history of the misshapen head? Neurosurgical Focus 2(2):Article 3, 1997

25. Muakkassa KF, Hoffman HJ, Hinton DR, et al: Lambdoid synostosis/ Part 2: Review of cases managed at The Hospital for Sick Children, 1972-1982. J Neurosurg 61:340-347, 1984

26. Persing JA, Delashaw JB, Jane JA, et al: Lambdoid synostosis: surgical considerations. Plast Reconstr Surg 81:852-860, 1988

27. Rekate HL: Diagnosis and management of plagiocephaly. Barrow Neurol Inst Q 2:69-75, 1986

28. Ripley CE, Pomatto J, Beals SP, et al: Treatment of positional plagiocephaly with dynamic orthotic cranioplasty. J Craniofacial Surg 5:150-160, 1994

29. Rout PG, Price C: Plagiocephaly. Br J Oral Surg 16:163-168, 1978

30. Sawin PD, Muhoned MG, Menezes AH: Quantitative analysis of cerebrospinal fluid spaces in children with occipital plagiocephaly. J Neurosurg 85:428-434, 1996

31. Shuper A, Merlob P, Grunebaum M, et al: The incidence of isolated craniosynostosis in the newborn infant. Am J Dis Child 139:85-86, 1985

32. Slate RK, Posnick JC, Armstrong DC, et al: Cervical spine subluxation associated with congenital muscular torticollis and craniofacial asymmetry. Plast Reconstr Surg 91:1187-1197, 1993

33. Tom LW, Handler SD, Wetmore RF, et al: The sternocleidomastoid tumor of infancy. Int J Pediatr Otorhinolaryngol 13:245-255, 1987

34. Tinuper P, Plazzi G, Provini F, et al: Facial asymmetry in partial epilepsies. Epilepsia 33:1097-1100, 1992

35. Wang FM: Plagiocephaly and torticollis. J Pediatrics 99:164, 1981 (Letter)

36. Watson GH: Relation between side of plagiocephaly, dislocation of hip, scoliosis, bat ears, and sternomastoid tumours. Arch Dis Child 46:203-210, 1971

37. Wilson ME, Hoxie J: Facial asymmetry in superior oblique muscle palsy. J Pediatr Ophthalmol Strab 30:315-318, 1993

38. Woon KY, Tan KL: Facial asymmetry and the sternomastoid tumour: a prospective study. J Singapore Paediatr Soc 19:255-261, 1977

Accepted in final form February 4, 1997. 
Address reprint requests to: Harold Rekate, M.D., Neurosurgical Associates, Ltd., 2910 North Third Avenue, Phoenix, Arizona, 85013. 